\title{
In vitro co-stimulation of anti-tumor activity by soluble B7 molecules
}

\author{
Wei He ${ }^{\star}$, Zhong-Bo Hu, Fang Liu, Xian-Qi Feng and Ping Zou ${ }^{凶}$ \\ Institute of Haematology, the Union Hospital, Tongji Medical College, Huazhong University of Science and

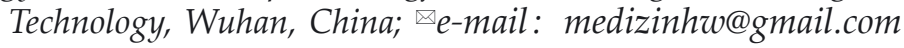

Received: 08 August, 2006; revised: 09 October, 2006; accepted: 17 October, 2006

available on-line: 22 November, 2006

\begin{abstract}
In order to investigate the anti-tumor activity of a soluble B7-1/immunoglobulin G fusion protein and explore an effective method to eliminate immune escape of tumor cells, a recombinant vector encoding this fusion protein was constructed and constitutively expressed in Chinese hamster ovary cells. After purification with protein $G$ affinity chromatography, the soluble fusion protein was tested for bioactivity. Results showed that the fusion protein could significantly increase the density of B7-1 molecules on WEHI-3 cells, a mouse leukemia cell line. Through allogeneic mixed lymphocyte tumor cultures, it was demonstrated that, with the presence of the first signal, it could also significantly enhance $T$ cell activation and killing activity against WEHI-3 cells and interleukin-2 secretion by activated mouse $T$ lymphocytes. The conclusion can be drawn that the soluble B7-IgG fusion protein has a potent capacity to generate or enhance anti-tumor immune response in vitro, and its clinical value deserves further investigation.
\end{abstract}

Keywords: gene fusion, recombinant fusion proteins, tumor escape, immunotherapy

\section{INTRODUCTION}

Generally, the normal anti-tumor immune response is initiated by occupancy of the T-cell receptor complex by tumor antigens processed and presented by APCs in association with the major histocompatibility complex, and by interaction of the adhesive molecules on APCs with their counterreceptors on $\mathrm{T}$ cells; these are respectively known as the first signal and the second signal (co-stimulatory signal) for T-cell activation. The first signal alone, lacking a co-stimulatory signal, leads to anergy or tolerance of tumor-specific T cells, and this has been established as the main mechanism by which some tumor cells escape host immunosurveillance (Whiteway et al., 2003), in that they can function as APCs. These tumor cells do not express B7 molecules on the surface of their membrane, or the expression is downregulated (Whiteway et al., 2003) by this means, they eliminate the critical co-stimulatory signal provided by the interaction of $\mathrm{B} 7 \mathrm{~mol}-$ ecules with their ligands on $\mathrm{T}$ cells (T-cell surface protein CD28 and/or CTLA-4). Any measures that effectively promote the expression of B7 molecules on the surface of tumor cells can at least partly restore their immunogenicity by signaling through the co-stimulatory pathway. It has been proved that transfection or transduction of the B7 gene into tumor cells can upregulate B7 molecule expression and thereby enhance anti-tumor immunity to some extent (Mutis et al., 1998). However, because of the low efficiency and time limitations of gene expression, the available B7 molecules are insufficient for full activation of tumor-specific $\mathrm{T}$ cells (Stein et al., 1991). The relative insufficiency of B7 gene transfection in tumor therapy can also be explained by its binding with CTLA-4 on activated T cells, which triggers a negative signal and facilitates immune tolerance to tumor cells (Bluestone, 1997). Here, we constructed a recombinant expressing vector by fusing a cDNA encoding the mouse IgG Fc fragment to a cDNA encoding the extracellular portion of mouse B7-1 antigen, which could generate a soluble fusion protein named B7-IgG. We presumed that this fu-

\footnotetext{
^Now at the Oncology and Haematology Center, the First Affiliated Hospital of Guangzhou Medical College, Guangzhou 510230, China.

Abbreviations: CTLA-4, cytotoxic T lymphocyte-associated antigen-4; FBS, fetal bovine serum; LDH, lactate dehydrogenase; $\mathrm{mAb}$, monoclonal antibody; MNC, mononuclear cells; HRP, horse radish peroxidase; PBS, phosphate-buffered saline; PHA, phytohemagglutinin; TAA, tumor-associated antigen.
} 
sion protein, though lacking the transmembrane and intracellular portions of the B7-1 molecule, would be sufficient for interaction with CD28 or CTLA-4 and would co-stimulate $\mathrm{T}$ lymphocytes. The Fc portion should permit its binding to the membrane of mouse leukemic cells via their highly expressed specific $\mathrm{Fc} \gamma$ receptors, which may upregulate expression of B7 molecules on leukemic cells and largely eliminate their immune escape. Also, we thought that the introduction of the soluble IgG Fc portion would give the molecule some new properties favoring the antitumor immune response.

\section{MATERIALS AND METHODS}

Construction of recombinant vector. The cDNA encoding the signal and extracellular domains of the mouse B7 molecule (termed cDNA-B7) was amplified from plasmid pcDNA/B7 containing the full-length cDNA of the mouse B7 molecule (a generous gift from Professor Qu Shen, Biochemistry Department, Tongji Medical College, Huazhong University of Science and Technology, China) with the following primers: forward 1: 5'-GCC GCT TAA GCT TCA TCT GAA GCT ATG GCT-3' and reverse 1: 5'-GCA CGA ATT CTG TGT TCT TGC TAT CAG G-3'. HindIII and EcoRI restriction enzyme sites were introduced into the above primers. The cDNA encoding the Fc portion (the hinge- $\mathrm{CH} 2-$ $\mathrm{CH} 3$ domains of mouse IgG1, termed cDNA-IgG) was obtained by reverse-transcription polymerase chain reaction (RT-PCR) with total RNA isolated from spleen cells of 8-week-old Balb/c mice (purchased from the Experimental Animal Center of Tongji Medical College). The primers were: forward 2: 5'-GCA CGA ATT CAG CAC CAA GGT GGA CAA G-3' and reverse 2: 5'-GCC ACT CGA GCC AAG GAC ACT GAG ATT A-3', into which EcoRI and XhoI restriction enzyme sites were introduced. Forward 1 included the initiation codon ATG and Reverse 2 included the termination codon TGA. To construct the pcDNA/B7-IgG recombinant vector, the plasmid pcDNA3.0 (kindly provided by Professor Guanxin Shen, Immunology Department, Tongji Medical College) and cDNA-IgG underwent double digestion with EcoRI and XhoI (Fermentas, Lithuania). After purification by phenol and chloroform, the cleavage products were ligated with $\mathrm{T} 4$ DNA ligase (Promega, USA) and then transformed into Escherichia coli strain DH5 $\alpha$ (generously provided by Professor Guanxin Shen). The transformants were singled out and plasmid DNA was isolated and identified on agarose gel by double digestion with EcoRI + XhoI and DNA sequencing. The correct recombinant was named pcDNA/IgG. Next, pcDNA/IgG and cDNA-B7 were subjected to double digestion with HindIII + EcoRI. After purification, ligation and transformation as described above, the products were verified on agarose gel by double digestion with HindIII + EcoRI, EcoRI + XhoI and HindIII + XhoI and DNA sequencing. The correct recombinant was named pcDNA/B7-IgG.

Cell transfection and expression. $\mathrm{CHO}$ cells were purchased from the China Center for Type Culture Collection (Wuhan, China) and maintained in DMEM/F12 medium (Gibco BRL, USA) containing $10 \%$ fetal bovine serum (FBS), $100 \mathrm{u} / \mathrm{ml}$ penicillin and $100 \mathrm{mg} / \mathrm{l}$ streptomycin at $37^{\circ} \mathrm{C}$ in a $5 \% \mathrm{CO}_{2}$ incubator. The pcDNA/B7-IgG plasmid was isolated and purified with a QIAGEN Maxi Plasmid Preparation Kit (Qiagen), and transfected into exponentially growing $\mathrm{CHO}$ cells with a liposome-mediated cell transfection kit (SuperFect Transfection Reagent, Qiagen), according to the manufacturer's instructions. The clones that survived G418 (Sigma, USA) selection (1-week incubation in DMEM/F12 medium containing $0.8 \mathrm{~g} / \mathrm{l} \mathrm{G} 418$ at $37^{\circ} \mathrm{C}$ ) were carefully isolated and transferred into DMEM/F12 medium containing $0.3 \mathrm{~g} / 1 \mathrm{G} 418$ for another 3-week incubation. A cell population with high constitutive expression of B7-IgG fusion protein was obtained and expanded, and some were then cryopreserved in liquid nitrogen. The control was the pcDNA3.0 plasmid. Supernatant of these surviving clones was collected and subjected to Western blotting under reducing conditions as previously described (Cai et al., 2002). The primary antibody was a goat polyclonal antibody raised against a peptide mapping at the amino terminus of CD80 of mouse origin (Santa Cruz, USA). The secondary antibody was HRP-conjugated rabbit anti-goat IgG (Zhongshan Biotech Co. Ltd., China). Luminol Reagent (Santa Cruz, USA) was used for enhanced chemiluminescence detection of Western blots. To perform dot ELISA, concentrated supernatants were dotted on nitrocellulose membrane (Sigma, USA) with serial dilution at 1:1, 1:2, 1:4, 1:8 and 1:16. A mouse IgG Fc fragment-specific HRPconjugated goat antibody (Biodesign, USA) was used for dot ELISA detection of B7-IgG as mentioned previously (Dalimi et al., 2004). The supernatant of the pcDNA3.0-transfected cell culture was regarded as negative control.

Purification of fusion protein. The $\mathrm{CHO}$ cells constitutively expressing the fusion protein were maintained in DMEM/F12 medium containing $0.3 \mathrm{~g} / \mathrm{l}$ G418. When they were at $30-40 \%$ confluence, the medium was removed and the cells were washed three times with serum-free DMEM/F12 medium; some OPTI-MEM reduced serum medium (Gibco BRL, USA) supplemented with $0.1 \mathrm{~g} / 1$ calcium chloride was then added and incubation continued. When these cells were at more than $90 \%$ confluence, the supernatant was harvested and centrifuged at $1000 \times$ 
$g$ for $10 \mathrm{~min}$ to eliminate cell debris. The fusion protein was isolated and purified from the supernatant by immune affinity chromatography with HiTrap Protein G affinity columns (Amersham Bioscience) according to the manufacturer's instructions. Briefly, $0.02 \mathrm{~mol} / \mathrm{l}$ sodium phosphate $(\mathrm{pH}$ 7.0) was prepared as the binding buffer and $0.1 \mathrm{~mol} / \mathrm{l}$ glycine/HCL $(\mathrm{pH}$ 2.7) as the elution buffer. The harvested supernatant $(200 \mathrm{ml})$ was concentrated by lyophilization and desalted by dialysis. The column was washed with 10 column volume of binding buffer, and a peristaltic pump was used to pass the sample through the column. After washing the column with 10 column volumes of binding buffer, the binding fusion protein was eluted with five column volumes of elution buffer. The eluted proteins were pooled and assayed by SDS/PAGE and Western blot as previously described, and then dialyzed overnight and sterilized by passing through $0.2 \mu \mathrm{m}$ syringe filter units. The concentration was determined by the UV absorbance at $280 \mathrm{~nm}$.

Effects of fusion protein on B7 density on leukemic cells. WEHI-3 cells were purchased from the Chinese Academy of Medical Science and were maintained in suspension culture in DMEM medium supplemented with $10 \%$ FBS, $100 \mathrm{u} / \mathrm{ml}$ penicillin and $100 \mathrm{mg} / \mathrm{l}$ streptomycin at $37^{\circ} \mathrm{C}$ in a $5 \% \mathrm{CO}_{2}$ incubator. Exponentially growing WEHI-3 cells were collected and washed three times with cold phosphate-buffered saline (PBS), and the cell density was adjusted to $1.0 \times 10^{6} / \mathrm{ml}$. After staining with a FITCconjugated anti-mouse CD80 monoclonal antibody (Southern Biotech, USA) and an R-phycoerythrinconjugated anti-mouse CD32 monoclonal antibody (Southern Biotech, USA), the cells were washed once with PBS and subjected to flow cytometry analysis. Irrelevant monoclonal antibodies of isotypes IgG1 and IgG2 (PharMingen, USA) served as negative controls. Next, WEHI-3 cells were preincubated at $4^{\circ} \mathrm{C}$ for $1 \mathrm{~h}$ with the fusion protein $\left(6 \mu \mathrm{g} / 10^{6}\right.$ cells (Zheng et al., 2001)) and washed extensively with PBS. After staining with FITC-anti-mouse CD80, the cells were subjected to flow cytometric analysis as described above.

T-cell proliferation assay and interleukin-2 secretion. Splenic cell suspension was obtained from a 6-week-old Balb/c mouse and mononuclear cells were isolated by Ficoll-Hypaque gradient centrifugation. Contaminating erythrocytes were removed by hypotonic lysis. Monocytes were removed by plastic adherence for $3 \mathrm{~h}$ in RMPI 1640 (Gibco BRL, USA) plus $10 \% \mathrm{FBS}$ at $37^{\circ} \mathrm{C}$ in $5 \% \mathrm{CO}_{2}$ incubator. $\mathrm{T}$ cells were enriched by passing through a nylon wool column and their purity was determined by flow cytometry with a FITC-conjugated anti-mouse CD3 monoclonal antibody (mAb, BioLegend). Purified anti-mouse CD3 mAb (17A2, BioLegend) was added at $25 \mu \mathrm{g} / \mathrm{ml}$ into wells in flat-bottom 96-well plates and incubated at $4^{\circ} \mathrm{C}$ overnight in coating buffer consisting of $50 \mathrm{mmol} / \mathrm{l} \mathrm{Tris} / \mathrm{HCl}(\mathrm{pH}$ 9.5) followed by extensive washing with RMPI 1640. T-cell proliferation reaction was assessed by incubating $1 \times 10^{5}$ purified $\mathrm{T}$ cells in flat-bottom 96-well plates in triplicate with or without B7-IgG protein $\left(6 \mu \mathrm{g} / 10^{6}\right.$ cells). $\mathrm{T}$ cell culture alone was regarded as a natural control. After 3-day incubation, these incubated $\mathrm{T}$ cells were pulsed with $1 \mu \mathrm{Ci}\left[{ }^{3} \mathrm{H}\right]$ thymidine per well for another 16 hours. The $\left[{ }^{3} \mathrm{H}\right] \mathrm{TdR}$ incorporation was measured by liquid scintillation counting to estimate the proliferation of lymphocytes. Likewise, the microculture system was repeated as mentioned above and the culture supernatant was aspirated at incubation day 2 and day 5 and assayed for interleukin-2 (IL-2) concentrations with mouse IL-2 ELISA kit (Jingmei Biotech, China) according to its handbook.

Allogeneic mixed lymphocyte tumor cultures. A splenic cell suspension was obtained from a 6-week-old Balb/c mouse and the MNCs were isolated by Ficoll-Hypaque gradient centrifugation. MNCs $\left(1.0 \times 10^{6}\right)$ were activated with $5 \mathrm{mg} / \mathrm{l} \mathrm{PHA}$ for 3 days in RMPI 1640 supplemented with 10\% FBS at $37^{\circ} \mathrm{C}$ in a $5 \% \mathrm{CO}_{2}$ incubator, followed by washing once, and cultured in RMPI 1640 medium for 1 day; these were regarded as responding cells (R). After inactivation by incubating $5 \times 10^{6}$ cells per $1 \mathrm{ml}$ DMEM medium in $1 \mathrm{~g} / \mathrm{l}$ mitomycin C (Sigma, USA) at $37^{\circ} \mathrm{C}$ in $5 \% \mathrm{CO}_{2}$ for $1 \mathrm{~h}$, WEHI-3 cells were preincubated in RMPI 1640 medium containing B7IgG protein $\left(6 \mu \mathrm{g} / 10^{6}\right.$ cells) or the medium alone (regarded as a control) at $4^{\circ} \mathrm{C}$ for $1 \mathrm{~h}$ and then carefully washed with PBS; these served as stimulating cells (S). For allogeneic mixed lymphocyte tumor culture, $5 \times 10^{5} / 100 \mu \mathrm{l}$ PHA-activated mouse MNCs (R) were carefully and completely mixed with $1.0 \times 10^{6} / 100$ $\mu$ stimulating cells $(S)$, resulting in an R:S ratio of $1: 2$, and then transferred to the microwells of flatbottom 96-well plates coated with anti-CD3 $\mathrm{mAb}$ as described above. They were incubated at $37^{\circ} \mathrm{C}$ in a $5 \% \mathrm{CO}_{2}$ incubator for 7 days, then the cytotoxic activity of the microcultures was determined by standard lactate dehydrogenase (LDH)-release assay. Briefly, exponentially growing WEHI-3 cells (not inactivated by mitomycin C) were used as target cells (T). The effector cells (E) and the target cells were carefully and completely mixed with an $\mathrm{E}: \mathrm{T}$ ratio of 25:1, added to 96-well flat-bottom plates (Corning, USA) with a final volume of $0.2 \mathrm{~mL}$ per well, and then incubated for $4 \mathrm{~h}$ in triplicates at $37^{\circ} \mathrm{C}$ in a $5 \%$ $\mathrm{CO}_{2}$ incubator. The maximum release (M) of LDH activity was determined by lysing the target cells with $1 \%$ NP40, and the spontaneous release (S) of LDH activity from target cells was detected by culturing WEHI-3 cells in culture medium alone. After incubation, the enzymatic reactions were carried out in the 
dark over $15 \mathrm{~min}$ as previously described (Konjevic et al., 1999). Release of LDH from tumor cells was expressed as absorbance at $490 \mathrm{~nm}$. The percentage of effector cell activity was calculated by the formula: cytotoxic activity $=(\mathrm{E}-\mathrm{S}) /(\mathrm{M}-\mathrm{S}) \times 100 \%$. All reagents were purchased from Sigma (St Louis, MO, USA).

Statistical analysis. All data were expressed as mean \pm S.D. and differences between groups were considered to be significant at $P<0.05$. Oneway analysis of variance was performed to estimate significant differences among groups by means of SPSS10.0, and vertical bar charts were created with SigmaPlot 8.0.

\section{RESULTS}

The recombinant vector was successfully constructed

By $1.5 \%$ agarose gel electrophoresis analysis, the sizes of cDNA-B7 (729 nucleotides, 243 amino acids) and cDNA-IgG (723 nucleotides, 241 amino acids) were confirmed to be about $750 \mathrm{bp}$, containing some attached bases. After digestion with $E c o R I+X h o I$, the recombinant pcDNA/IgG released two DNA fragments; one was about $750 \mathrm{bp}$, which should be cDNA-IgG and the other was about 5.4 $\mathrm{kb}$, which was the remaining bases of pcDNA3.0. Various double digestions, including HindIII + EcoRI, EcoRI + XhoI and HindIII + XhoI, were performed to confirm that the recombinant pcDNA/B7-IgG contained the two target cDNA fragments in tandem. DNA sequencing showed that the cDNA sequences of the two fragments were identical to the reported sequences, and they had been cloned in tandem into pcDNA3.0 with a correct open reading frame. The conclusion could be drawn that the recombinant vector of $\mathrm{pcDNA} / \mathrm{B} 7-\mathrm{IgG}$ had been constructed successfully.

Constitutive expression, identification and purification of B7-IgG fusion protein. As shown in Fig. $1 \mathrm{~A}$ and $1 \mathrm{~B}$, an antigen was present in the supernatant of the G418-selected $\mathrm{CHO}$ cell population that could be recognized not only by a specific antibody targeted at the extracellular portion of B7-1, but also by an $\operatorname{IgG}$ Fc fragment-specific antibody. Its molecular mass was 70-100 kDa. However, none of the controls contained this antigen. Theoretically, the calculated molecular mass of this fusion protein monomer is about $55 \mathrm{kDa}$, as it contains about 484 amino acids. However, if glycosylation is taken into account, it should be a little greater. Hence, we considered this to be the B7-IgG fusion protein that we had constructed. Figure 1C shows that the purified fusion protein migrated at approx. 66-97 kDa in
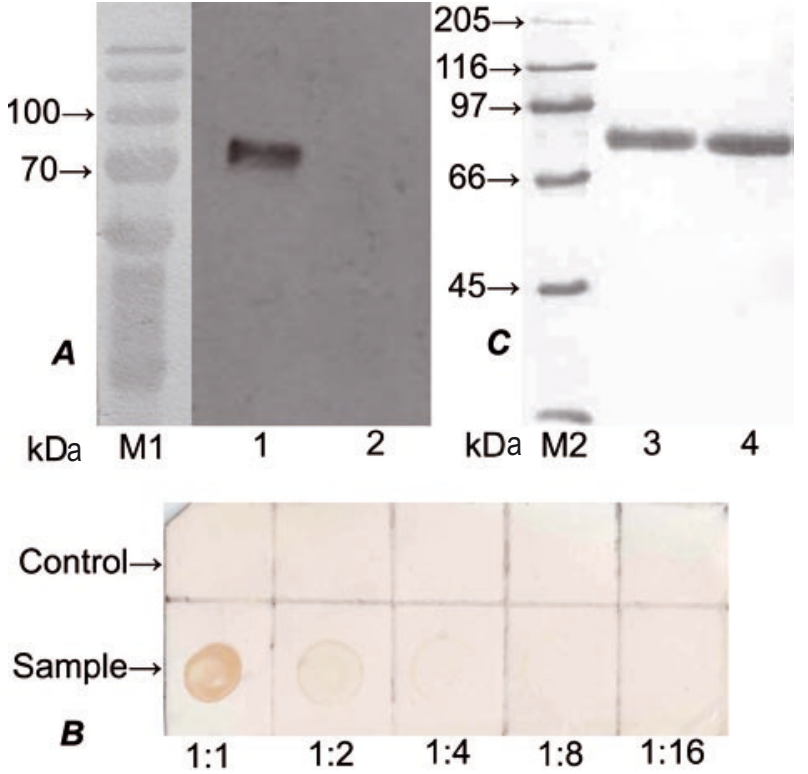

Figure 1. Expression, identification and purification of B7-IgG fusion protein.

A. Western blotting analysis. M1: prestained protein ladder 10-180 $\mathrm{kDa}$; lane 1: fusion protein recognized by a specific antibody targeted at extracellular portion of B71; lane 2: control; B. Dot ELISA analysis with the IgG FC fragment-specific antibody. C. SDS/PAGE analysis of purified protein. M2: high molecular mass protein marker; lanes 3 and 4: samples 3 and 4 .

SDS/PAGE under reducing conditions. Western blotting analysis showed a specific band at the expected site (not shown), which coincided with what was proven previously. According to these data combined with the absorbance of the protein solution at $280 \mathrm{~nm}$, we measured the concentration of the fusion protein to be about $0.1 \mathrm{mg} / \mathrm{ml}$ and the purity more than $90 \%$. In all, $20 \mathrm{mg}$ of the fusion protein was obtained from $200 \mathrm{ml}$ of cell culture supernatant.

Density of B7-1 molecules on leukemic cells was increased by fusion protein

As shown in Fig. 2, the very low expression of B7-1 molecules and the very high expression of CD32 molecules on WEHI-3 cells indicated that the WEHI-3 cell line was suitable for our experimental model. After preincubation with the fusion protein, WEHI-3 cells showed a significant $(P<0.01)$ increase in the density of B7-1 molecules on their membrane.

\section{Co-stimulation of T-cell proliferation and enhance- ment of IL-2 secretion}

Figure $3 \mathrm{~A}$ and $3 \mathrm{~B}$ show that, in the presence of the first signal imitated by anti-CD3 $\mathrm{mAb}, \mathrm{B} 7-$ IgG protein could dramatically co-stimulate purified mouse $\mathrm{T}$ cells to proliferate by providing them with the second signal; the first signal alone could not. These co-stimulated $\mathrm{T}$ cells secreted much more 

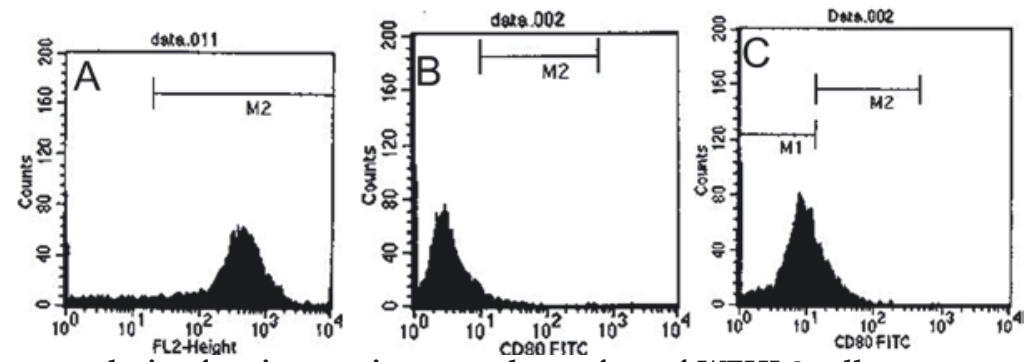

Figure 2. Flow cytometry analysis of various antigens on the surface of WEHI-3 cells.

CD32 expression rate (A) was about $(89.48 \pm 3.05) \%(n=5)$. Before treatment with fusion protein, CD80 density (B) on the membrane of WEHI-3 cells was about $(5.06 \pm 0.41) \%(n=5)$, but after preincubation with fusion protein, CD80 density (C) was significantly upregulated to $(27.36 \pm 2.15) \%(\mathrm{n}=4, P<0.01)$.

IL-2 than those primed by anti-CD3 mAb alone to further enhance this effect, although the increase in IL-2 secretion could be completely accounted for by the increase in T-cell proliferation. It is worth noting that, after a 5-day incubation, $\mathrm{T}$ cells co-stimulated by the fusion protein together with anti-CD3 could still maintain a high IL-2 level $(P>0.05)$, but other T cells could not $(P<0.05)$. We presumed that this was because the fusion protein could promote continu-

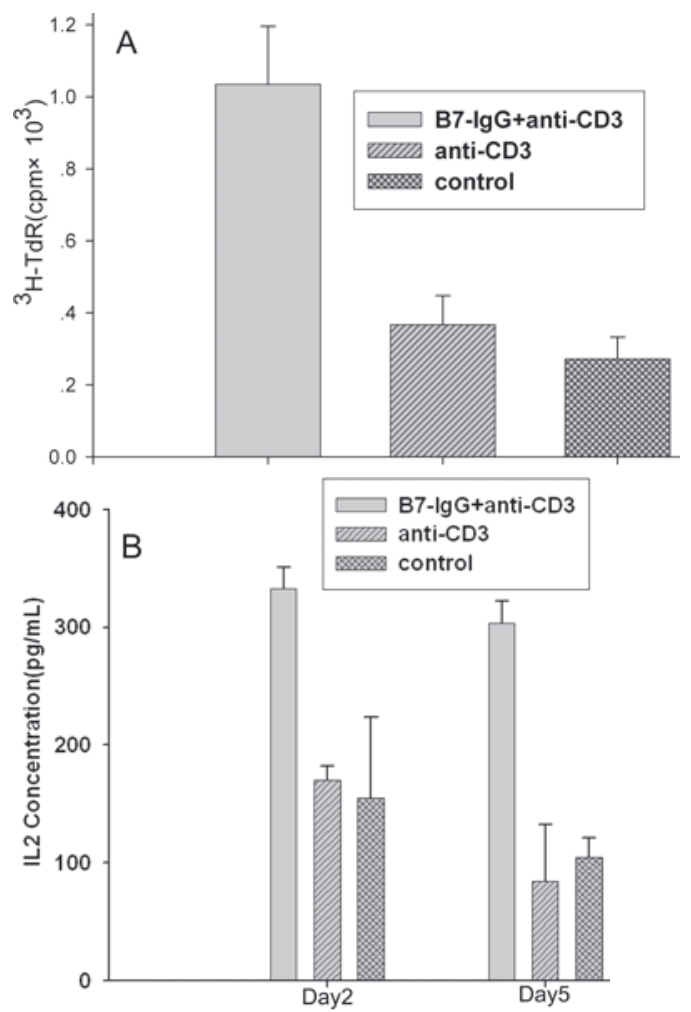

Figure 3. Co-stimulation of $\mathrm{T}$ cells by anti-CD3 mAb together with B7-IgG fusion protein or alone, and IL-2 secretion.

A. The measured $\left[{ }^{3} \mathrm{H}\right] \mathrm{TdR}$ incorporation is the average of five independent experiments, and with co-stimulation by B7-IgG+anti-CD3, it was significantly increased (compared with that of the control, $P<0.05$ ); however, that of anti-CD3 alone was not $(P<0.05)$. B. IL-2 concentration is shown as the average of five independent experiments. Compared with the control, the IL-2 concentration increase after co-stimulation by B7-IgG and anti-CD3 was statistically significant $(P<0.05)$, but that with anti-CD3 alone was not $(P<0.05)$. All data are represented as mean \pm S.D. ous secretion of IL-2 for at least 5 days and maintain activation and proliferation of $\mathrm{T}$ lymphocytes.

\section{Enhanced cytotoxicity against allogeneic tumor cells}

The lytic activity of lymphocytes against WEHI-3 cells is positively associated with LDH release. The results (shown in Table 1) suggested that B7-IgG-treated tumor cells, which resulted in pronounced upregulation of B7 expression on the cells, had a significantly increased susceptibility to killing by primed lymphocytes. Treatment without the fusion protein did not cause pronounced cytotoxicity against tumor cells. This observation showed the ability of the B7-IgG fusion protein to co-stimulate lymphocytes and enhance their cytotoxicity against target cells.

\section{DISCUSSION}

B7 is a member of the Ig superfamily, which is mainly expressed on APCs. B7-1 and B7-2 (CD86) are two important molecules of the family. By binding with their two main ligands, CD28 and/or CTLA4 , they provide the most important co-stimulatory signal for the $\mathrm{T}$ cell proliferative response and induction of effector function. However, ligation of B7 and CTLA-4 generates a negative signal for immune response, and only the interaction of B7 and CD28 can induce proliferation and lymphokine secretion. If the antigen is presented to APCs without the costimulatory signal, antigen-specific $\mathrm{T}$ cells become non-responsive. Many tumor cells, especially AML cells, can function as APCs, but impaired expression of $\mathrm{B} 7$ on their membrane makes it very easy

Table 1. Lymphocyte activity against allogeneic tumor cells

\begin{tabular}{lll}
\hline Group & $\mathrm{n}$ & Cytotoxicity $(\%)$ \\
\hline Preincubation & 6 & $63.16^{*}$ \\
Control & 6 & 19.30 \\
\hline
\end{tabular}

${ }^{*}$ Compared with control group, $P<0.05$. 
for them to escape host immune system surveillance and attack (Whiteway et al., 2003). Although CD86 is a more important source of the co-stimulatory signal, maybe because its intracellular portion is longer than that of CD80, many data show there is much more seriously impaired expression of CD80 than CD86 on tumor cells (Matsumoto \& Anasetti, 1999) and transfection with the CD80 gene, which results in expression of CD80 molecules, could more effectively protect established animal models from tumor cell rechallenge and relapse from minimal residual disease (Matulonis et al., 1996). With a view to increasing the expression of CD80 molecules on tumor cells, we constructed a B7-IgG fusion protein containing the signal peptide and two IgG-like domains of the extracellular portion of the CD80 antigen. Our data give strong support to the hypothesis that, in the presence of the first signal simulated by anti-CD3 $\mathrm{mAb}$ in vitro, a soluble B7-IgG fusion protein including only the extracellular portion of the CD80 molecule could, as in transfection with fulllength CD80 cDNA, boost the expression of CD80 on tumor cells and co-stimulate naïve $\mathrm{T}$ cells to proliferate, secrete IL-2 and thereby enhance their killing activity against tumor cells. Early in 1999, an investigation was reported (Moro et al., 1999) that a biotinylated B7-IgG fusion protein could induce potent anti-cancer immunity. But this approach was based on a sequential incubation of cultured tumor cells with biotinylated antibodies specific for a membrane tumor-associated antigen (TAA), avidin and biotinylated soluble B7-IgG fusion protein, which is a multistep procedure not suitable for clinical application. The rationale underlying our approach is the binding with high affinity of the Fc fragment of the fusion protein to Fc gamma receptors highly expressed on some tumor cells like AML. This binding occurs in physiological conditions.

As a new type of cluster of differentiation (CD)/immunoglobulin G (IgG) fusion protein, B7IgG has many advantages over B7 gene transfection or transduction in terms of tumor immunotherapy.

Firstly, it is soluble and can be produced in bulk in vitro by genetic engineering. Secondly, introduction of the $\mathrm{CH}$ domain of the heavy chain into the fusion protein gives it a longer half-life in vivo and makes it more suitable for in vivo use. Thirdly, there are investigations showing that $\mathrm{F}_{\mathrm{C}}$ gamma receptors are highly expressed on nearly all AML cells (Notter et al., 1993), but have a very restricted pattern of expression in normal cells (Olweus et al., 1995). Our results prove high-level expression of $\mathrm{CD} 32$, the mouse IgG2b/1 Fc receptor on WEHI-3 cells. It has been established (Notter et al., 1993) that AML cells whose Fc gamma receptors were bound by an antiCD3 mAb, like B7-IgG, were preferentially lysed in vitro by cytotoxic $\mathrm{T}$ lymphocytes, and most of the normal cells were spared. Taken together, these results suggest that the fusion protein has greater effects on tumor cells than on normal cells. Fourthly, by means of its IgG Fc portion, the membranebound fusion protein can enrich co-stimulatory molecules in the local microenvironment around tumor cells and magnify co-stimulatory signals, which can greatly improve anti-tumor immunity. Lastly, unlike B7 gene transfection, soluble B7-IgG can bind with high affinity to CTLA-4 on activated T cells to block negative signals triggered by it, thereby further enhancing the anti-tumor activity.

The potency of the B7-IgG fusion protein in stimulating anti-tumor immune responses suggests that it may be useful in optimizing anticancer vaccines and has clinical potential as therapy for oncology and for other clinical indications. As described previously (Oh et al., 2003), increasing levels of costimulation, using a triad of costimulatory molecules-CD80, ICAM-1 and LFA-3-in conjunction with a vaccine, can selectively induce CTLs skewed toward higher avidity and more effective at killing tumor cells. Soluble B7-IgG fusion protein can also be used as an effective molecular adjuvant in tumor immune therapy. Intramuscular gene transfer of B7IgG fusion gene induce potent anti-tumor immunity as an adjuvant for DNA vaccination (Zhou et al., 2003). Leukemia may be cured by intravenous administration of the fusion protein. Indeed, the combination of conventional chemotherapy and systemic immunotherapy with soluble B7-IgG fusion protein could synergize to induce curative T-cell-dependent antitumor responses and immunologic memory in murine leukemia and lymphoma models (Runyon et al., 2001). More recently, this fusion protein was shown to be able to induce complete regression of some solid tumors after intravenous injection if combined with regulatory $\mathrm{T}$ cell depletion (Liu et al., 2005). But the efficacy lasts for only a short time because of the protein's short half-life. In addition, the safety of intravenous injection of a high dose of this fusion protein remains undetermined in spite of a research in which maximum $500 \mu \mathrm{g}$ protein was injected to mice and did not show any in vivo toxicity (Runyon et al., 2001). We constructed this recombinant expressing vector with a view to eradicating tumor cells by intratumor injection of this vector or infusion of fusion gene-modified $\mathrm{T}$ cells from donors into leukemia recipients. If so, systemic exposure to these co-stimulatory molecules and consequent in vivo toxicity will be minimized and the efficacy will last for much longer because of the persistence of gene expression.

In summary, targeting of tumor cells with the B7-IgG fusion protein is a new strategy of immunotherapy for tumors including leukemias and some solid tumors, with the potential for further preclini- 
cal trials and future clinical application. However, this is a manmade molecule, and it remains undetermined how this soluble molecule induces potent anti-tumor immunity; further studies are needed. From our data, it is uncertain whether this fusion molecule can interplay with its ligands in vivo and promote a tumor-specific immune response. Also, further investigation is needed to determine whether the fusion protein can do harm to the human body.

\section{Acknowledgements}

This project was supported by the National Natural Science Foundation of China (Grant No. 30240022).

We thank Professor Qingfen Li and Professor Guanxin Shen for helpful discussion during the course of this work. We thank Technicians Wei Feng, Xiaodan Jiang, and Ping Xiong for their kind help in RT-PCR, Western blotting and protein purification.

Also, we thank the Nuclear Medicine Department of the Union Hospital, Tongji Medical College, Huazhong University of Science and Technology (China) for technical support for radioactive isotope assay.

\section{REFERENCES}

Bluestone JA (1997) Is CTLA-4 a master switch for peripheral T cell tolerance? J Immunol 158: 1989-1993.

Cai SB, Ma QJ, Yu XJ, Dang GT, Ma DL (2002) Expression of human VEGF $_{121}$ cDNA in mouse bone marrow stroma cells. Chin Med J 115: 914-918.

Dalimi A, Hadighi R, Madani R (2004) Partially purified fraction (PPF) antigen from adult Fasciola gigantica for the serodiagnosis of human fascioliasis using Dot-ELISA technique. Ann Saudi Med 24: 18-20.

Konjevic G, Jurisic V, Banicevic B, Spuzic I (1999) The difference in NK-cell activity between patients with nonHodgkin's lymphomas and Hodgkin's disease. $\mathrm{Br} J$ Haematol 104: 144-151.

Liu A, Hu P, Khawli LA, Epstein AL (2005) Combination B7-Fc fusion protein treatment and Treg cell depletion therapy. Clin Cancer Res 11: 8492-8502.

Matsumoto K, Anasetti C (1999) The role of T cell costimulation by CD80 in the initiation and maintenance of the immune response to human leukemia. Leuk Lymphoma 35: 427-435.
Matulonis U, Dosiou C, Freeman G, Lamont C, Mauch P, Nadler LM, Griffin JD (1996) B7-1 is superior to B7-2 costimulation in the induction and maintenance of $\mathrm{T}$ cell-mediated antileukemia immunity. Further evidence that B7-1 and B7-2 are functionally distinct. J Immunol 156: $1126-1131$.

Moro M, Gasparri AM, Pagano S, Bellone M, Tornaghi P, Veglia F, Corti A, Casorati G, Dellabona P (1999) Induction of therapeutic T-cell immunity by tumor targeting with soluble recombinant B7-immunoglobulin costimulatory molecules. Cancer Res 59: 2650-2656.

Mutis T, Schrama E, Melief CJ, Goulmy El (1998) CD80transfected acute myeloid leukemia cells induce primary allogeneic $\mathrm{T}$-cell responses directed at patient specific minor histocompatibility antigens and leukemia-associated antigens. Blood 92: 1677-1684.

Notter M, Ludwig WD, Bremer S, Thiel E (1993) Selective targeting of human lymphokine-activated killer cells by CD3 monoclonal antibody against the interferoninducible high affinity $\mathrm{Fc}_{\mathrm{C}}$ gamma RI receptor (CD64) on autologous acute myeloid leukemic blast cells. Blood 82: 3113-3124.

Oh S, Hodge JW, Ahlers JD, Burke DS, Schlom J, Berzofsky JA (2003) Selective induction of high avidity CTL by altering the balance of signals from antigen presenting cells. J Immunol 170: 2523-2530.

Olweus J, Lund-Johansen F, Terstappen LW (1995) CD64/ Fc gamma RI is a granulo-monocytic lineage marker on CD34+ hematopoietic progenitor cells. Blood 85: 2402-2413.

Runyon K, Lee K, Zuberek K, Collins M, Leonard JP, Dunussi-Joannopoulos K (2001) The combination of chemotherapy and systemic immunotherapy with soluble B7-immunoglobulin G leads to cure of murine leukemia and lymphoma and demonstration of tumor-specific memory responses. Blood 97: 2420-2426.

Stein H, Herbst H, Anagnostopoulos I, Niedobitek G, Dallenbach F, Kratzsch HC (1991) The nature of Hodgkin and Reed-Sternberg cells, their association with EBV, and their relationship to anaplastic large-cell lymphoma. Ann Oncol 2 (Suppl 2): 33-38.

Whiteway A, Corbett T, Anderson R, Macdonald I, Prentice HG (2003) Expression of co-stimulatory molecules on acute myeloid leukemia blasts may effect duration of first remission. Br J Haematol 120: 442-451.

Zheng G, Chen A, Sterner RE, Zhang PJ, Pan T, Kiyatkin $\mathrm{N}$, Tykocinski ML (2001) Induction of antitumor immunity via intratumoral tetra-costimulator protein transfer. Cancer Res 61: 8127-8134.

Zhou ZF, Peretz Y, Chang Y, Miao DS, Li XY, Prud'homme GJ (2003) Intramuscular gene transfer of soluble B7.1/ $\operatorname{IgG(1)}$ fusion cDNA induces potent antitumor immunity as an adjuvant for DNA vaccination. Cancer Gene Ther 10: 491-499. 